\title{
1 Potential high value conjugated linolenic acids (CLnA) in melon seed waste
}

2

3 Supplementary information

4

5 Dong Hao Wang ${ }^{\mathrm{a}, \mathrm{b}}$, Zhen Wang ${ }^{\mathrm{a}, \mathrm{b}}$, Kim Phuong Le ${ }^{\mathrm{a}}$, J. Raven Cortright ${ }^{\mathrm{a}}$, Hui Gyu Park ${ }^{\mathrm{a}}$, Herbert

6 J. Tobias ${ }^{a *}$, J. Thomas Brenna ${ }^{a, b, c *}$

7

8 a. Dell Pediatric Research Institute, University of Texas at Austin, 1400 Barbara Jordan Blvd,

9 Austin, TX, United States

10 b. Department of Food Science, Cornell University, Ithaca, NY, United States

11 c. Division of Nutritional Sciences, Cornell University, thaca, NY, United States

12

$13{ }^{*}$ Corresponding author present address. Dell Pediatric Research Institute, University of Texas

14 at Austin, 1400 Barbara Jordan Blvd, Austin, TX 78723. Tel: 607-218-8812

15 E-mail address:tbrenna@utexas.edu (J. T. Brenna); herbert.tobias@austin.utexas.edu (H.

16 Tobias)

17 
SupplementaryTable 1. Concentration (Mean $\pm \mathrm{SD}, \mathrm{mg} / \mathrm{g}$ of seed kernels) of conjugated linolenic acids in various

SupplementaryFigure 2. Supplementarychromatograms to Figure 1 together showing the elution order of CLnA

32 Supplementary Figure 3-1. MRM chromatograms of cantaloupe CLnAdiagnostic ions used to calculate the relative

SupplementaryFigure 3-3. Structures and diagnostic ions of 7 major CLnA in cantaloupe as labeled in

Supplementary Figure 4. Calibration curve for CLnA quantification. (a)Calibration curve of $[\mathrm{M}+1]$ and (b) calibration curve of diagnostic ions. $[M+1]$ ion is used when the analyte is well resolved; diagnostic ions are used

43 Supplementary Figure 6. Chromatograms of coeluting CLnAisomers by diagnostic ions. (a) Reconstructed chromatograms of a diagnostic ions of pomegranate seed extract (mainlypunicic acid or 9Z,11E,13Z-18:3) and (b) 


\section{Supplementary Tables}

49 Supplementary Table 1. Concentration (Mean $\pm \mathrm{SD}, \mathrm{mg} / \mathrm{g}$ of seed kernels) of conjugated linolenic acids in various

50 fruit seeds.

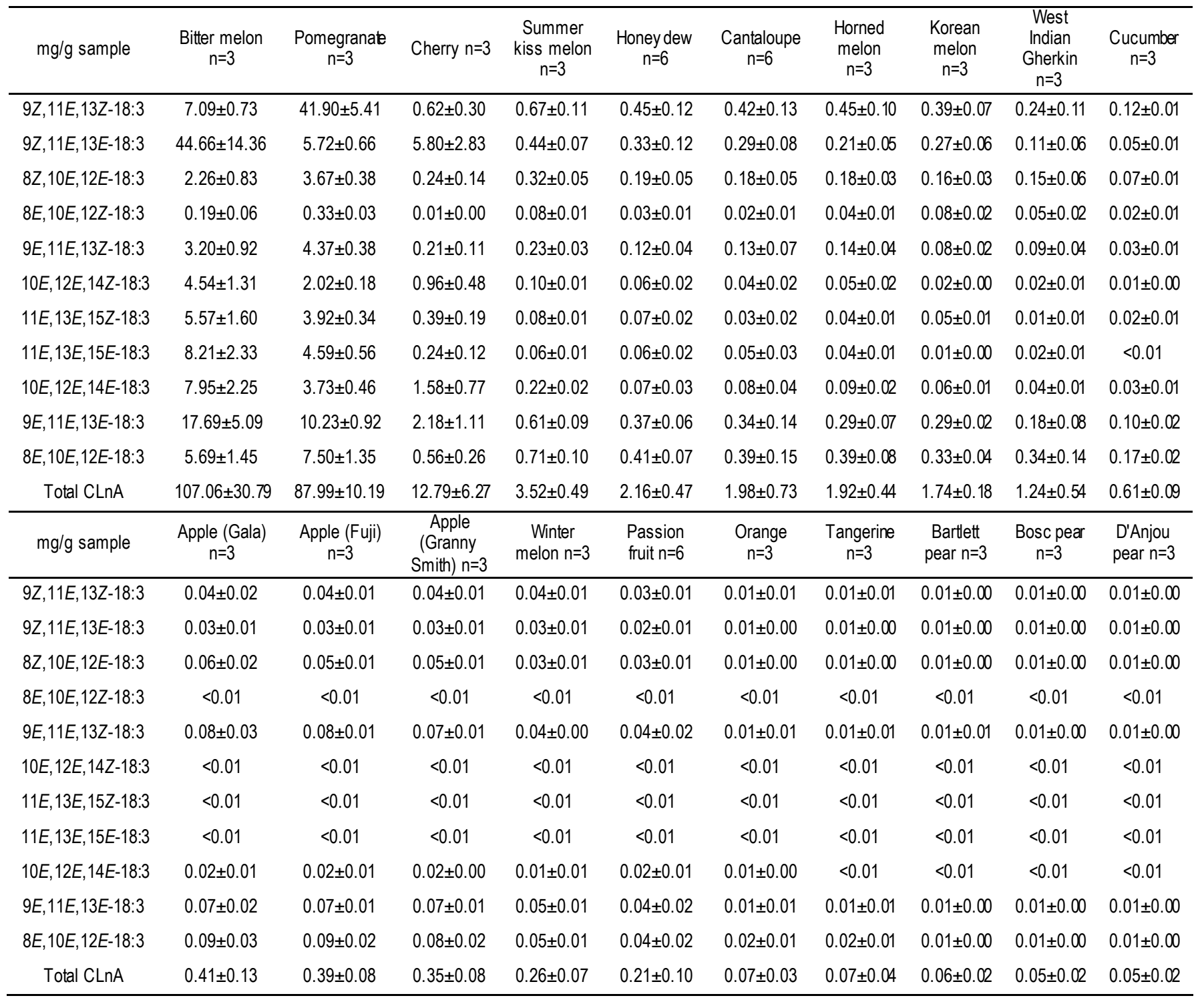

51

52 Note: All replicates are number of biological samples. 
53 Supplementary Table 2. Scientific and common names of all fruits analyzed for CLnA content.

\begin{tabular}{|c|c|c|c|}
\hline \multicolumn{2}{|c|}{ Fruit seeds with CLnA } & \multicolumn{2}{|c|}{ Fruit seeds with no detectable CLnA } \\
\hline Scientific name & Common name & Scientific name & Common name \\
\hline Benincasa hispida & Winter melon* & Ananas comosus & Pineapple \\
\hline Citrus sinensis & Orange* & Prunus persica & White peach \\
\hline Citrus tangerina & Tangerine ${ }^{*}$ & Mangifera indica & Mango \\
\hline Cucumis anguria & West Indian gherkin* & Dimocarpus longan & Longan \\
\hline Cucumis melo & Summer kiss melon* & Artocarpus heterophyllus & Jackfruit \\
\hline Cucumis melo & Honeydew* & Litchi chinensis & Lychee \\
\hline Cucumis melo & Cantaloupe* & Vitis vinifera & Grape \\
\hline Cucumis melo & Korean melon* & P. domestica & Black Plum \\
\hline Cucumis metuliferus & Horned melon* & Prunus persica & Yellow peach \\
\hline Cucumis sativus & Cucumber* & Garcinia mangostana & Mangosteen \\
\hline Malus pumila & Apple $(F u j i)^{*}$ & Opuntia spp. & Prickly pears \\
\hline Malus pumila & Apple (Granny Smith)* & Capsicum annuum & Red bell pepper \\
\hline Malus pumila & Apple $(\text { Gala })^{*}$ & Nephelium lappaceum & Rambutan \\
\hline Momordica charantia & Bitter melon & Psidium guajava & Guava \\
\hline Passiflora edulis & Passion fruitt & Annona cherimola & Cherimoya \\
\hline Prunus avium & Cherry & Prunus spp. & Plumcot \\
\hline Punica granatum & Pomegranate & P. domestica & Green plum \\
\hline Pyrus communis & Pear $(\text { Bosc })^{*}$ & P. domestica & Red plum \\
\hline Pyrus communis & Pear (D'Anjou) ${ }^{*}$ & Carica papaya & Papaya \\
\hline \multirow[t]{11}{*}{ Pyrus communis } & Pear (Barttett) $)^{*}$ & Prunus persica var. platycarpa & Donut peach \\
\hline & & Ziziphus jujuba & Jujube \\
\hline & & Actinidia deliciosa & Kiwi \\
\hline & & Rubus spp. & Blackberry \\
\hline & & Hylocereus undatus & Dragonfruit \\
\hline & & Persea americana & Avocado \\
\hline & & Capsicum annuum & Green bell pepper \\
\hline & & Raphanus raphanistrum & Radish \\
\hline & & Spinacia oleracea & Spinach \\
\hline & & Solanum lycopersicum & Roma tomato \\
\hline & & Solanum lycopersicum & Hamson tomato \\
\hline
\end{tabular}

54

${ }^{*}$ These are new sources of CLnA which have never been reported previously. 
56 SupplementaryTable 3. Determination of seed kernel proportion of whole honeydew, cherryand apple.

\begin{tabular}{cccc}
\hline & $\begin{array}{c}\text { Honeydew }(\mathrm{n}=6 \\
\text { biological samples) }\end{array}$ & $\begin{array}{c}\text { cherry }(\mathrm{n}=3 \\
\text { biological samples) }\end{array}$ & $\begin{array}{c}\text { Apple }(\mathrm{n}=3 \\
\text { biological samples })\end{array}$ \\
\hline Fruit, g & 1544 & 5.8 & 544 \\
Seeds, g & 39.8 & 0.34 & 0.288 \\
Weight seed/weight fruit & 0.0258 & 0.0594 & 0.0005 \\
Fraction of fruit nonedible & 0.25 & 0.07 & 0.07 \\
One seed, g & 0.06 & 0.34 & 0.07 \\
One seed kernel, g & 0.03 & 0.11 & 0.04 \\
Weight kernel/weight seed & 0.56 & 0.31 & 0.64 \\
Weight kernel/weight fruit & 0.0146 & 0.0187 & 0.0001 \\
\hline
\end{tabular}

57 
58 SupplementaryTable 4. Calculations and estimation of total available CLnA $(\mathrm{Kg})$ in US fruit waste steam.

\begin{tabular}{cccccc}
\hline & Cherry & Honeydew & Cantaloupe & Apple & Pear \\
\hline Total CLnA( $\mu \mathrm{g} / \mathrm{g}$ seeds) & 12794 & 2156 & 1977 & 383 & 54 \\
Pounds per capita a & 2.1 & 1.6 & 7.1 & 18.7 & 4.4 \\
US Annual Production, Kg $^{\mathrm{b}}$ & $314,618,111$ & $235,228,494$ & $1,046,766,798$ & $2,744,822,489$ & $642,467,824$ \\
Weight kernel/weight fruitc $^{\text {c }}$ & 0.0187 & 0.0146 & 0.0146 & 0.0001 & 0.0001 \\
Waste of seeds, Kg & $5,881,912$ & $3,423,569$ & $15,234,882$ & 213,094 & 49,878 \\
CLnAUS Fruit Waste,Kg & 75,253 & 7,381 & 30,119 & 82 & 3 \\
\hline
\end{tabular}

59

60 a Data for y ear 2016 was obtained from USDA ERS. https://www.ers. usda.gov/data-products/food-availability-per-capita-data-system/

61 b US population $324,118,787$ in 2016 was used for the calculations.

62 c Determined by w eight for cherry, honeydew and apple in Supplementary Table 3. Cantaloupeand pear are similar to honeydew and apple and the same ratio 63 was used respectively. 
64 Supplementary Table 5. Calculations of market value of CLnA based on current conjugated linoleic acid (CLA) retail 65 price.

\begin{tabular}{cccc}
\hline Brand Selling CLA supplements & USD & $\mathrm{g}$ & USD/g \\
\hline A & 29.99 & 90 & 0.33 \\
B & 13.38 & 50 & 0.27 \\
C & 14.99 & 144 & 0.10 \\
D & 8.99 & 72 & 0.12 \\
E & 17.99 & 144 & 0.12 \\
F & 14.99 & 90 & 0.17 \\
G & 21.80 & 214 & 0.10 \\
H & 19.94 & 225 & 0.09 \\
I & 16.95 & 108 & 0.16 \\
J & 8.99 & 68 & 0.13 \\
\hline Mean value (USD/g) & & & 0.16 \\
Muskmelon seeds CLnAUS total,Kg & & & 37,500 \\
CLnApotential revenue (Million USD) & & & 6.00 \\
\hline
\end{tabular}

66

67 Note: Retail price was listed online as for June 4, 2019. 
SupplementaryTable 6. MS/MS mass table of $y$-linolenic acid and 7 characteristic CLnA.

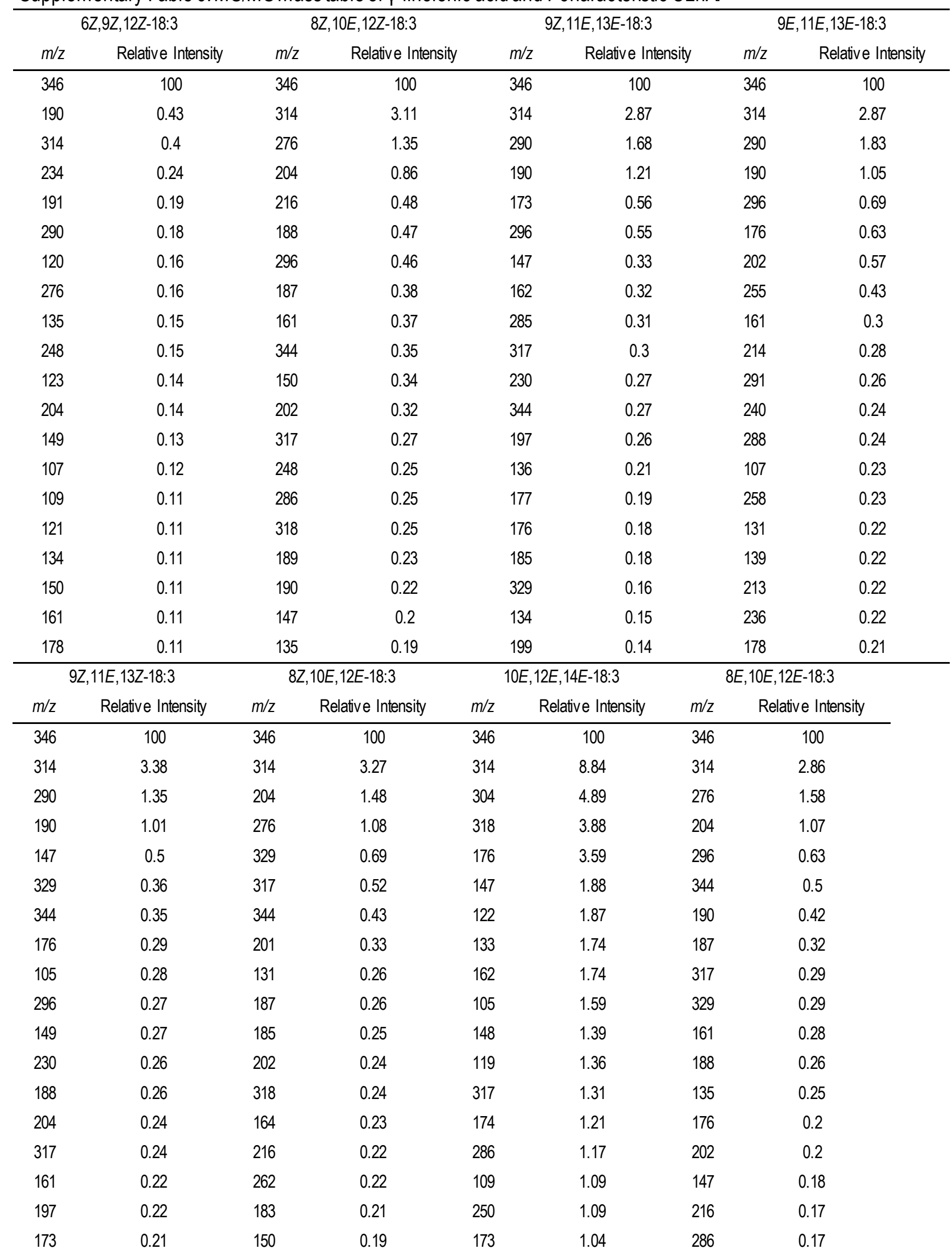


267

$\begin{array}{lll}0.21 & 133 & 0.18\end{array}$

0.17

146

1.03

131

0.16

69

185

100

121

1.01

159

0.16 


\section{Supplementary Figures}

71 Supplementary Figure 1. CIMS/MS spectra of authentic standards. (a) Jacaric acid or 8Z,10E,12Z-18:3;

72 (b) a-eleostearic acid or 9Z,11E,13E-18:3; (c)Calendic acid or 8E,10E,12Z; (d) Catalpic acid or 9E,11E,13Z-18:3; (e)

73 B-eleostearic acid or $9 E, 11 E, 13 E-18: 3$.
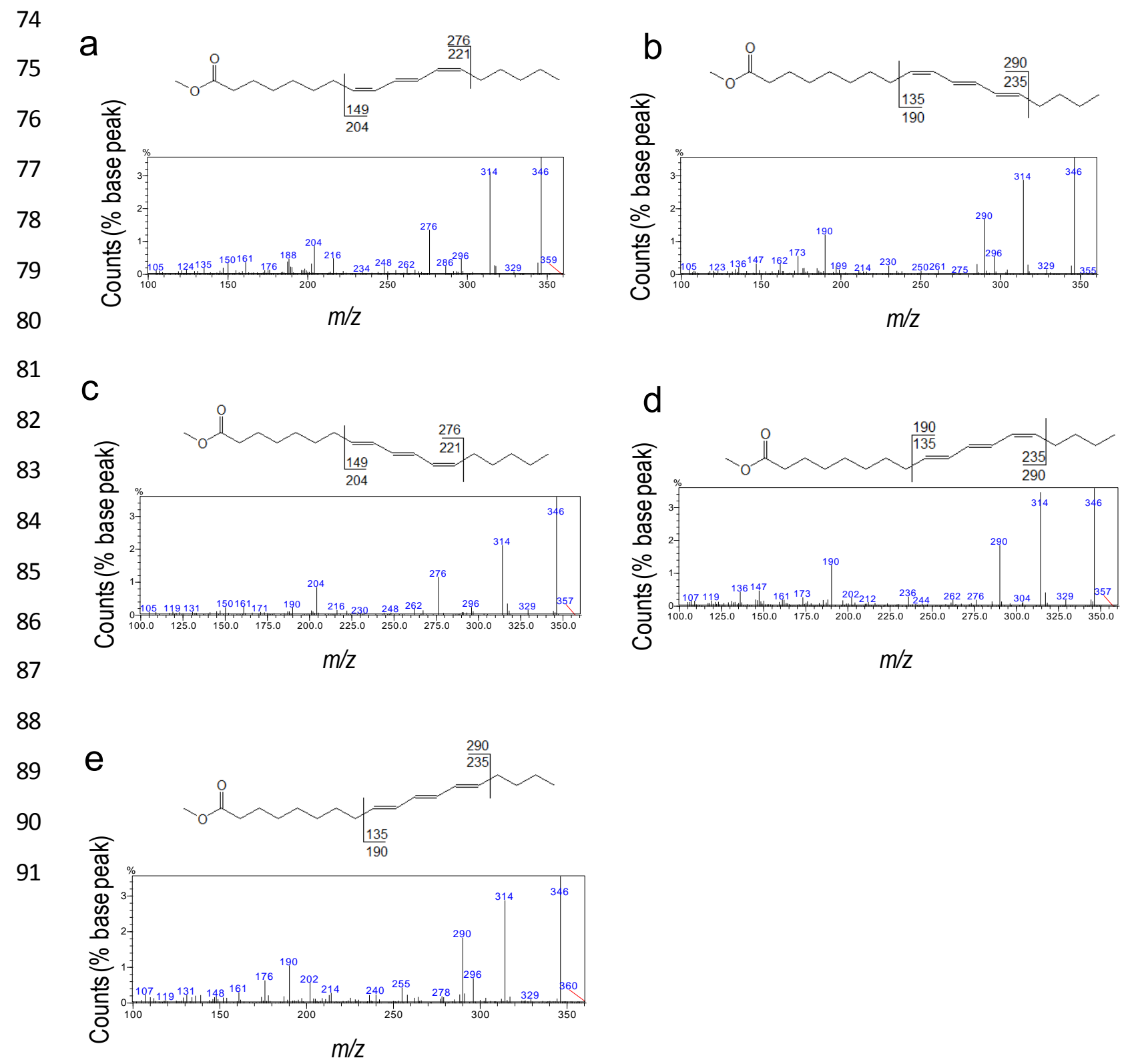
92 Supplementary Figure 2. Supplementarychromatograms to Figure 1 together showing the elution order of CLnA on a 93 BPX-70 column.
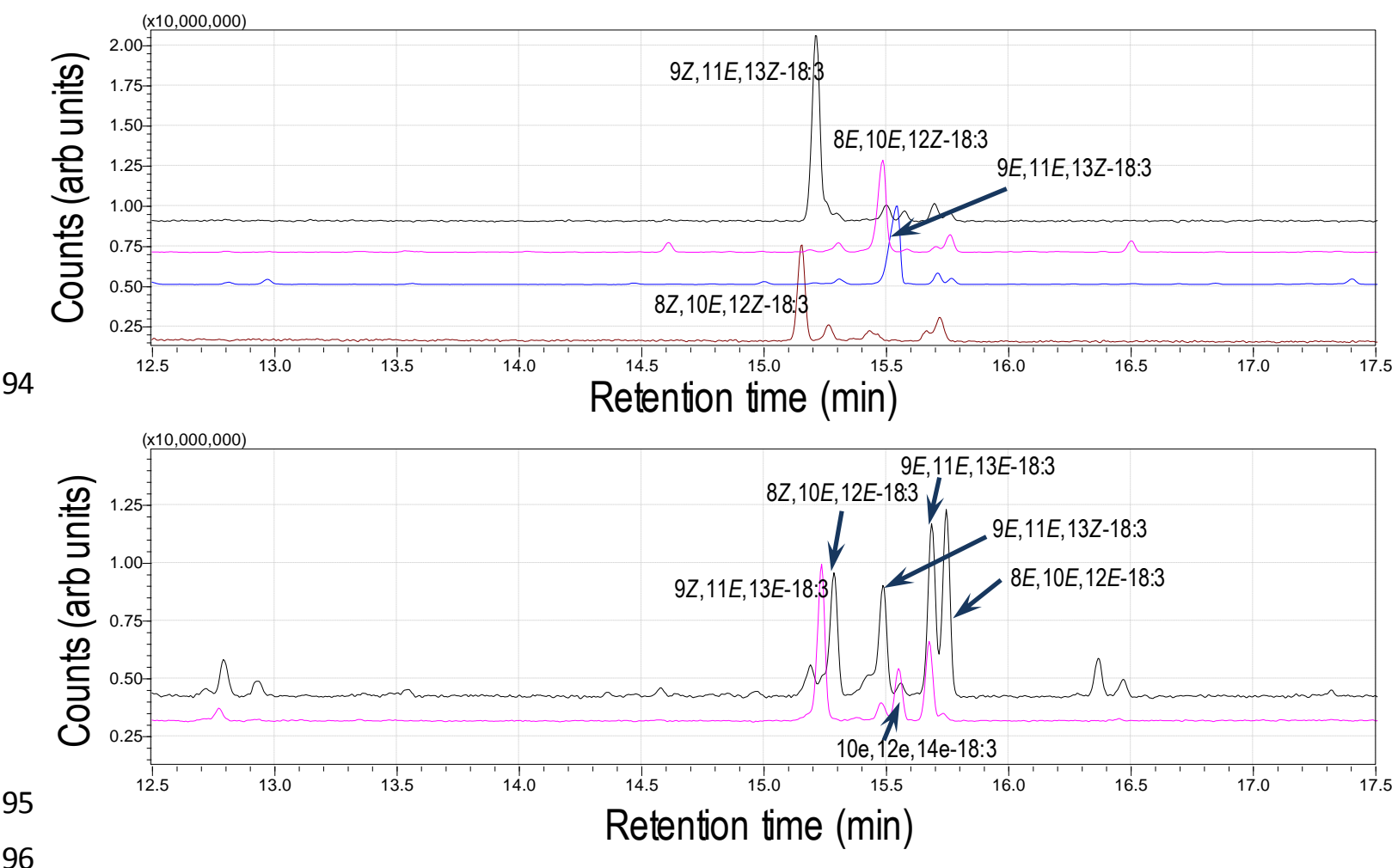

96

97 Order: cis $(Z)$,trans $(E)$,cis $(Z)<$ cis,trans,trans < trans,trans,cis < trans, trans,trans $8 Z, 10 E, 12 Z<9 Z, 11 E, 13 Z<10 Z, 12 E, 14 Z$ (predicted)

98 $10 Z, 12 E, 14 E$ (predicted) $<9 Z, 11 E, 13 E<8 Z, 10 E, 12 E$

$998 E, 10 E, 12 Z<9 E, 11 E, 13 Z<10 E, 12 E, 14 Z<11 E, 13 E, 15 Z$ (coeluted as one peak) $11 E, 13 E, 15 E<10 E, 12 E, 14 E<9 E, 11 E, 13 E<8 E, 10 E, 12 E$ 
100 SupplementaryFigure 3-1. MRM chromatograms of cantaloupe CLnAdiagnostic ions used to calculate the relative 101 abundance of unresolved CLnA isomers.

102

103

104

105

106

107

108

109

110

111

112

113

114

115

116
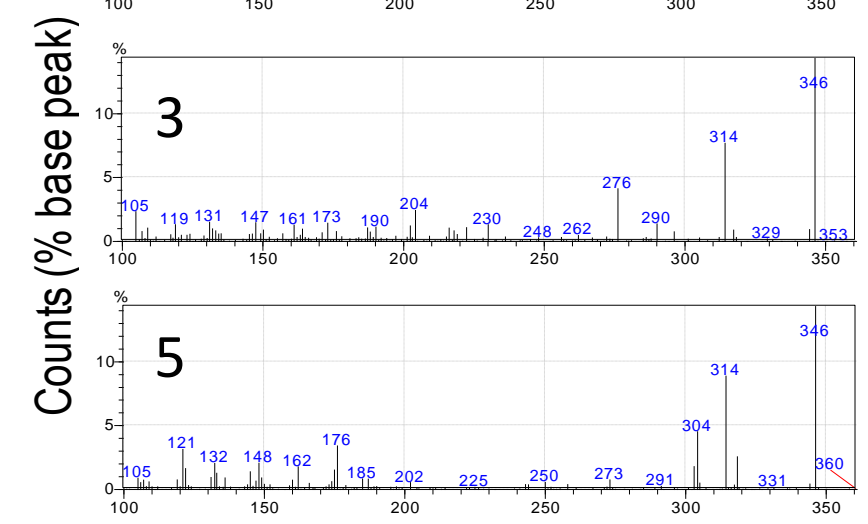

117

118

119
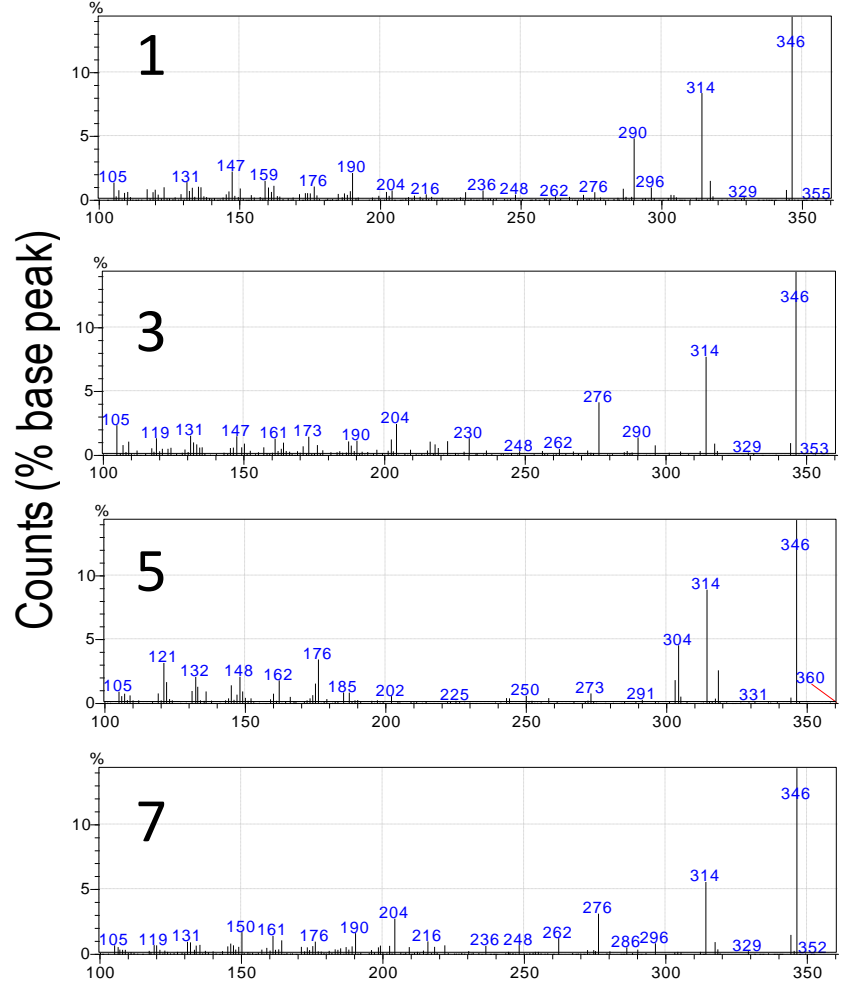

\section{6}

$m / z$

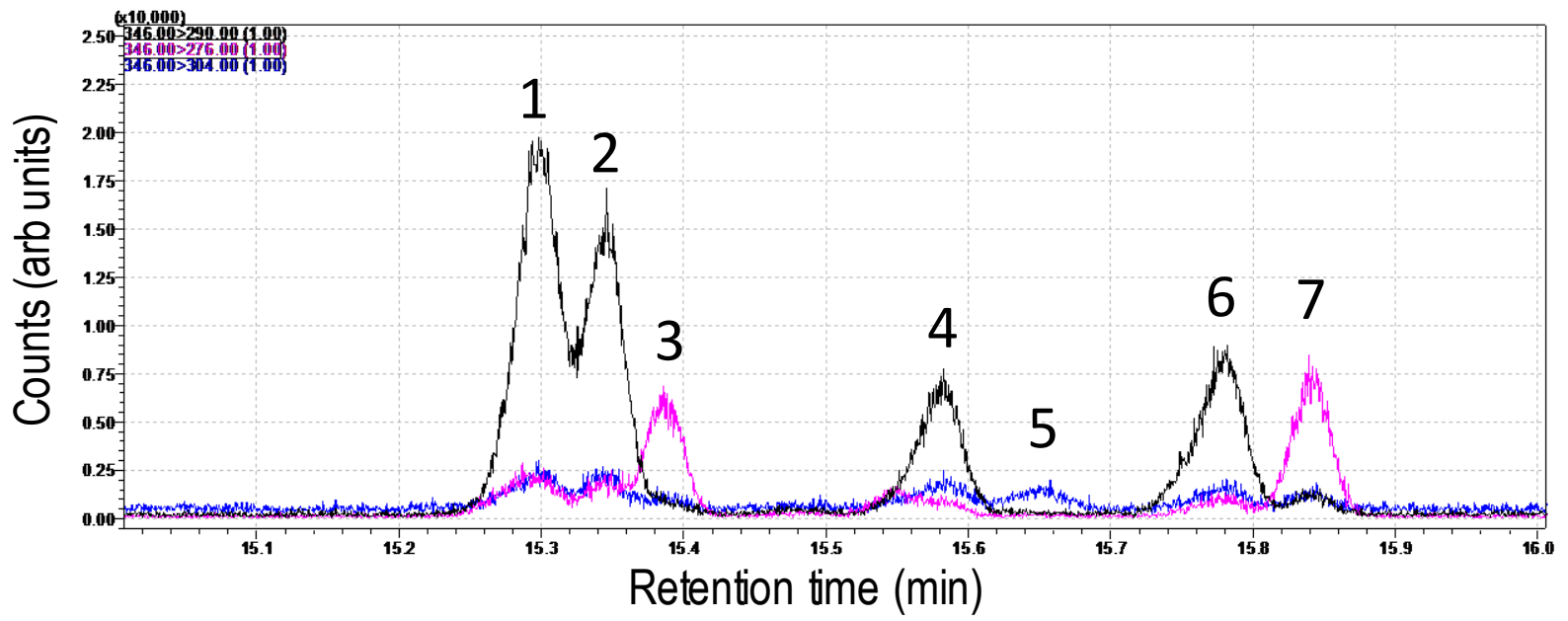

SupplementaryFigure 3-2. CIMS/MS spectra of 7 major CLnA in cantaloupe as labeled in Supplementary Figure 3-1.
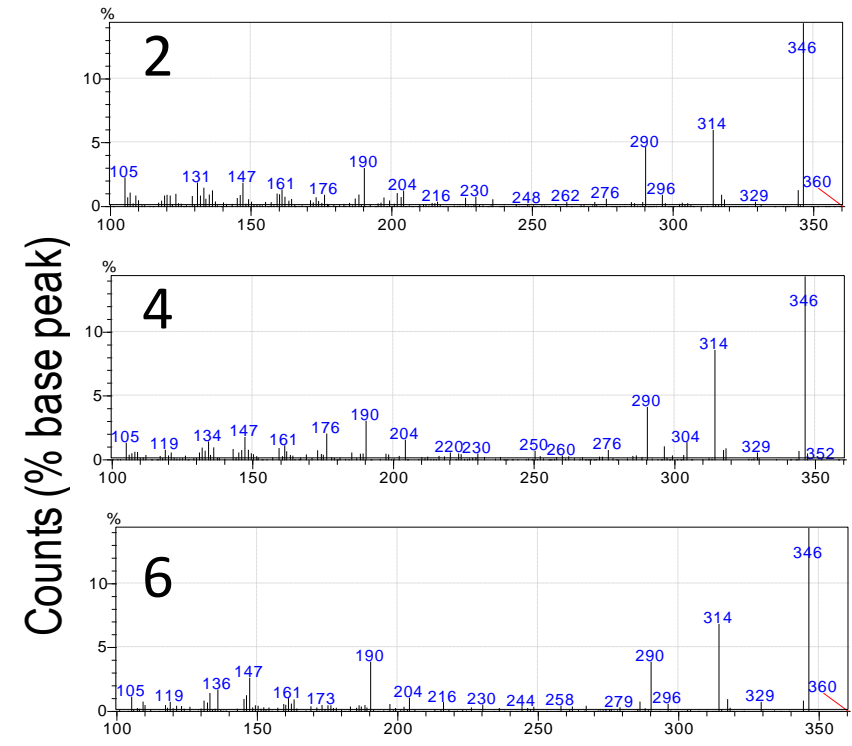

$m / z$ 
120 SupplementaryFigure 3-3. Structures and diagnostic ions of 7 major CLnA in cantaloupe as labeled in 121 SupplementaryFigure 3-1.

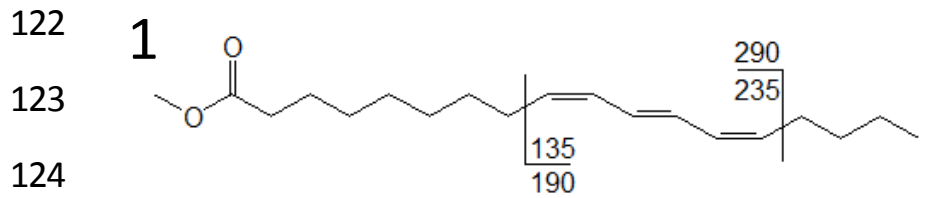

125

126

127

128

129

130

131

132

133

134

135

136

1377

138

139

140

141

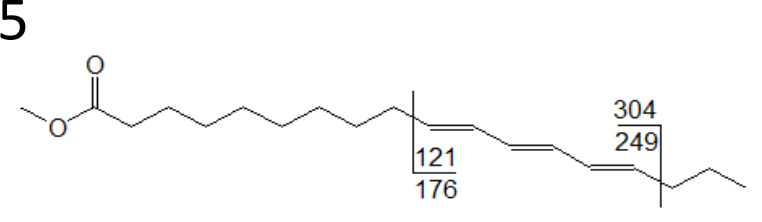

$10 E, 12 E, 14 E-18: 3$

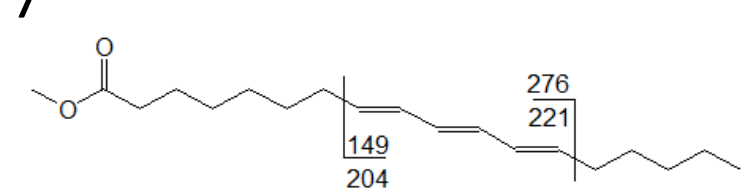

$8 E, 10 E, 12 E-18: 3$
2

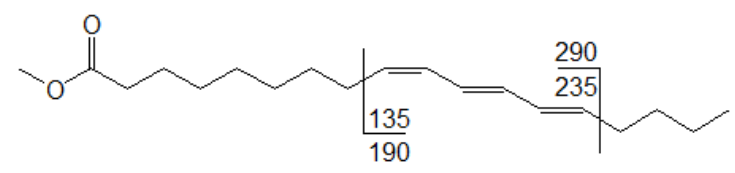

$9 Z, 11 E, 13 E-18: 3$

4

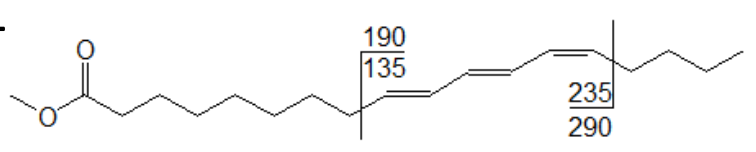

$9 E, 11 E, 13 Z-18: 3$

6

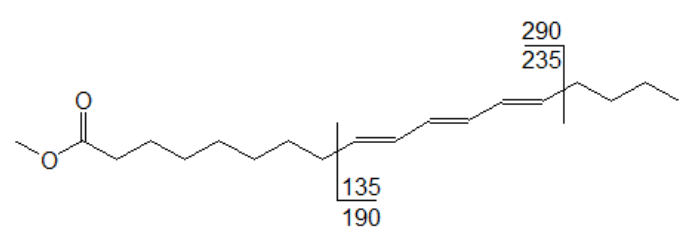

$9 E, 11 E, 13 E-18: 3$ 
142 Supplementary Figure 4. Calibration curve for CLnA quantification. (a) Calibration curve of [M+1] 143 and (b) calibration curve of diagnostic ions. $[\mathrm{M}+1]$ ion is used when the analyte is well resolved;

144 diagnostic ions are used when CLnA isomers cannot be resolved by GC alone .

145

146

a

147

148

149

150

151

152

153

154

155

156

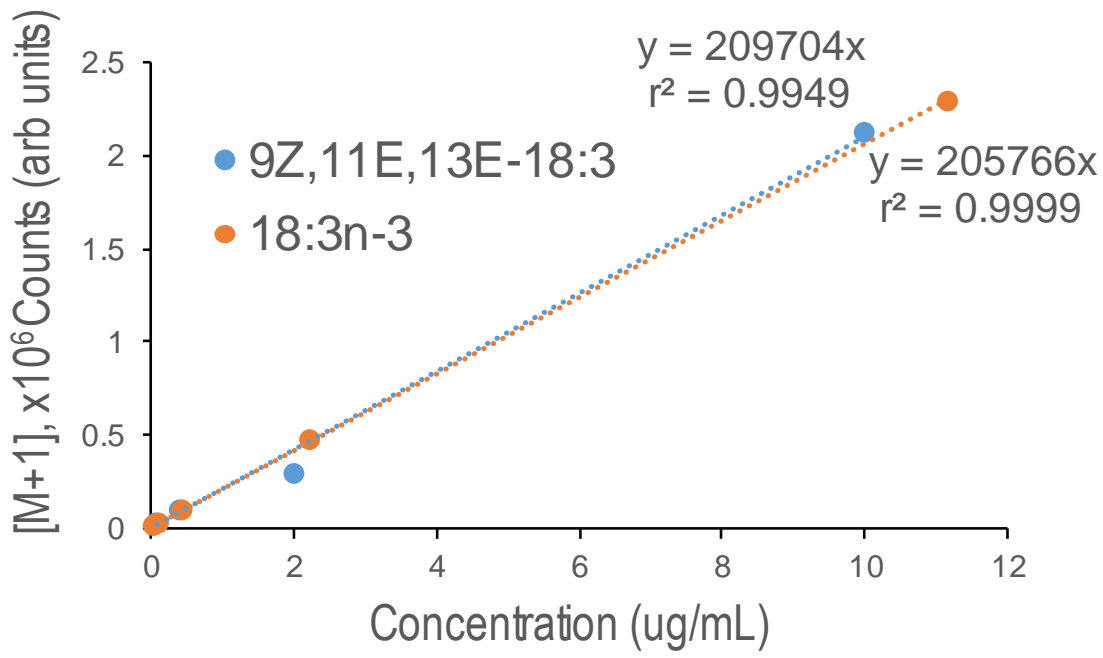

157

158

b

159

160

161

162

163

164

165

166

167

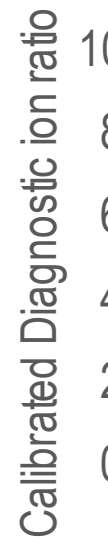

168

- $\omega / \omega$ ratio

- a/a ratio

- Mean $a / a \& \omega / \omega$

- $y=x$

$y=1.03 x$

$r^{2}=0.9951$

.......

$a$ 
172 SupplementaryFigure 5. MRM chromatogram resolving and quantifying 9E,11E,13Z-18:3 and 8E,10E,12Z-18:3 by a 173 diagnostic ions, $\mathrm{m} / \mathrm{z} 290 \& 276$, respectively.

174

175

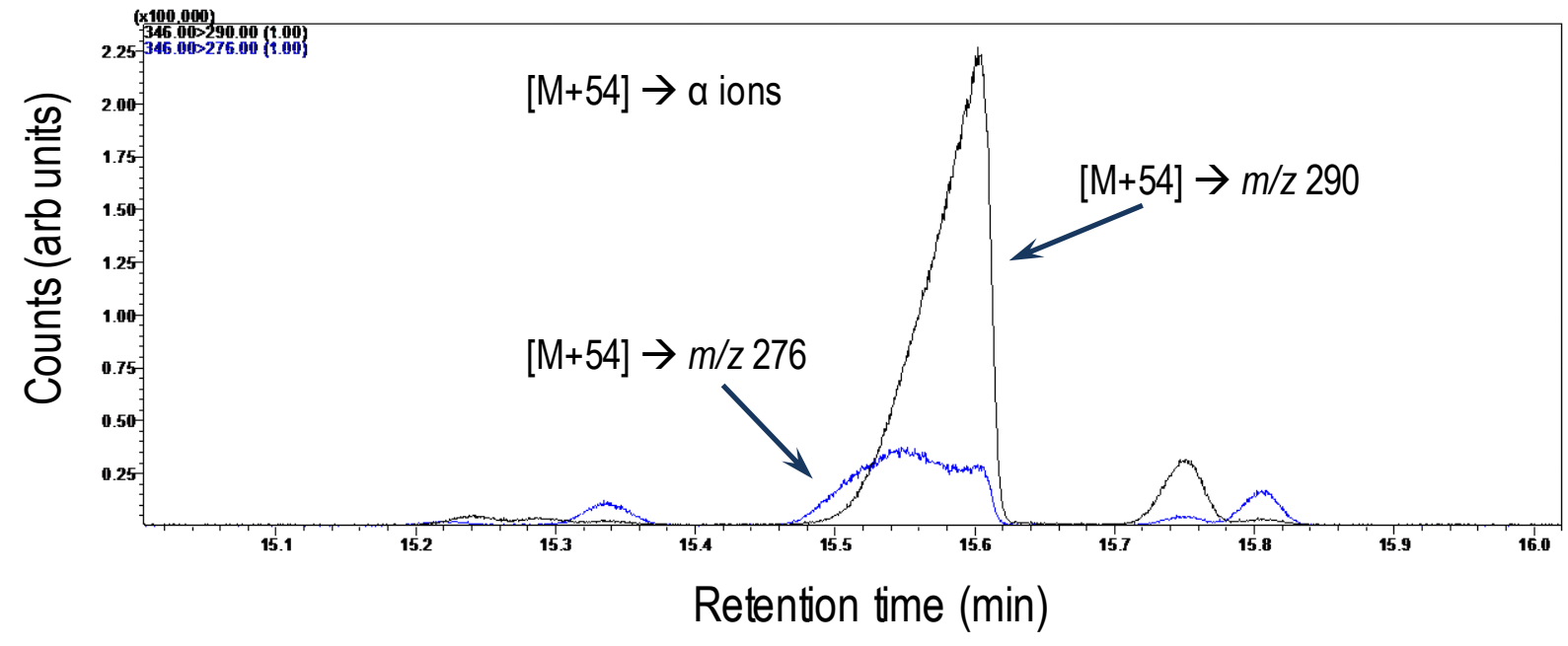


176 SupplementaryFigure 6. Chromatograms of coeluting CLnAisomers by diagnostic ions. (a) Reconstructed

177 chromatograms of a diagnostic ions of pomegranate seed extract (mainlypunicic acid or 9Z,11E,13Z-18:3) and (b)

178 the same sample spiked with jacaric acid (8Z,10E,12Z-18:3) at one third amount of punicic acid.

179

181

a

180

182

183

184

185

186

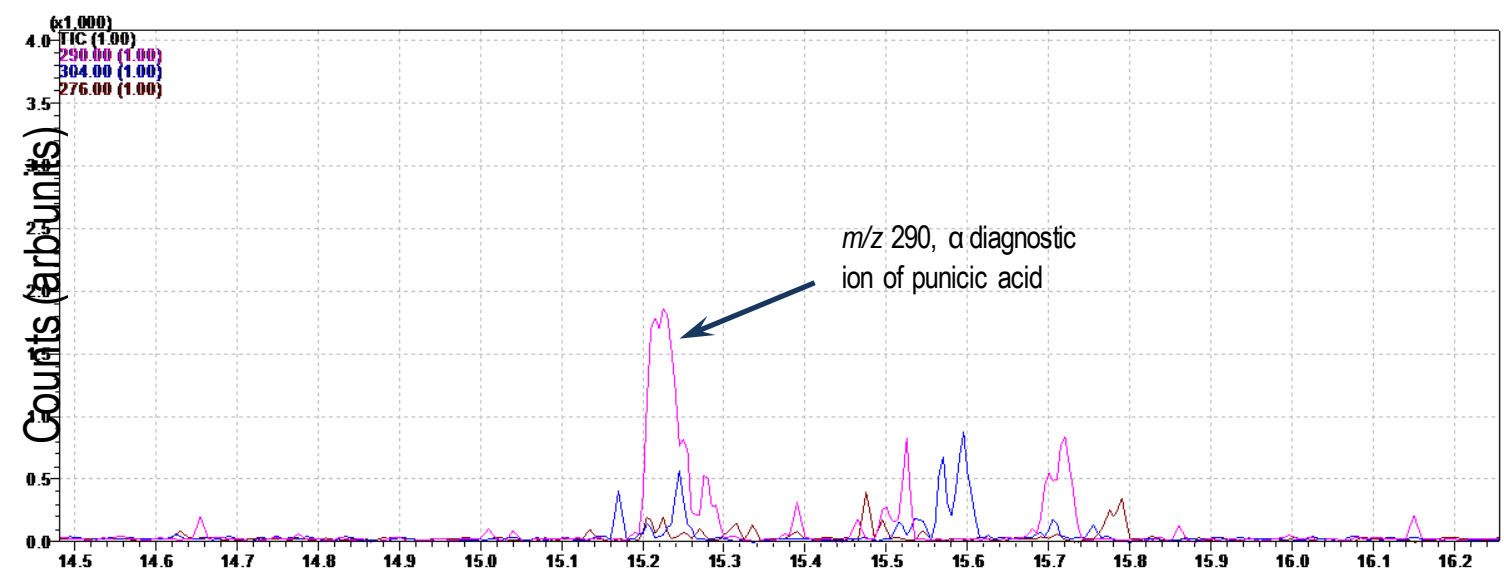

187

$18 \mathrm{~b}$

189

190

191

192

193

194

195

196

Retention time (min)

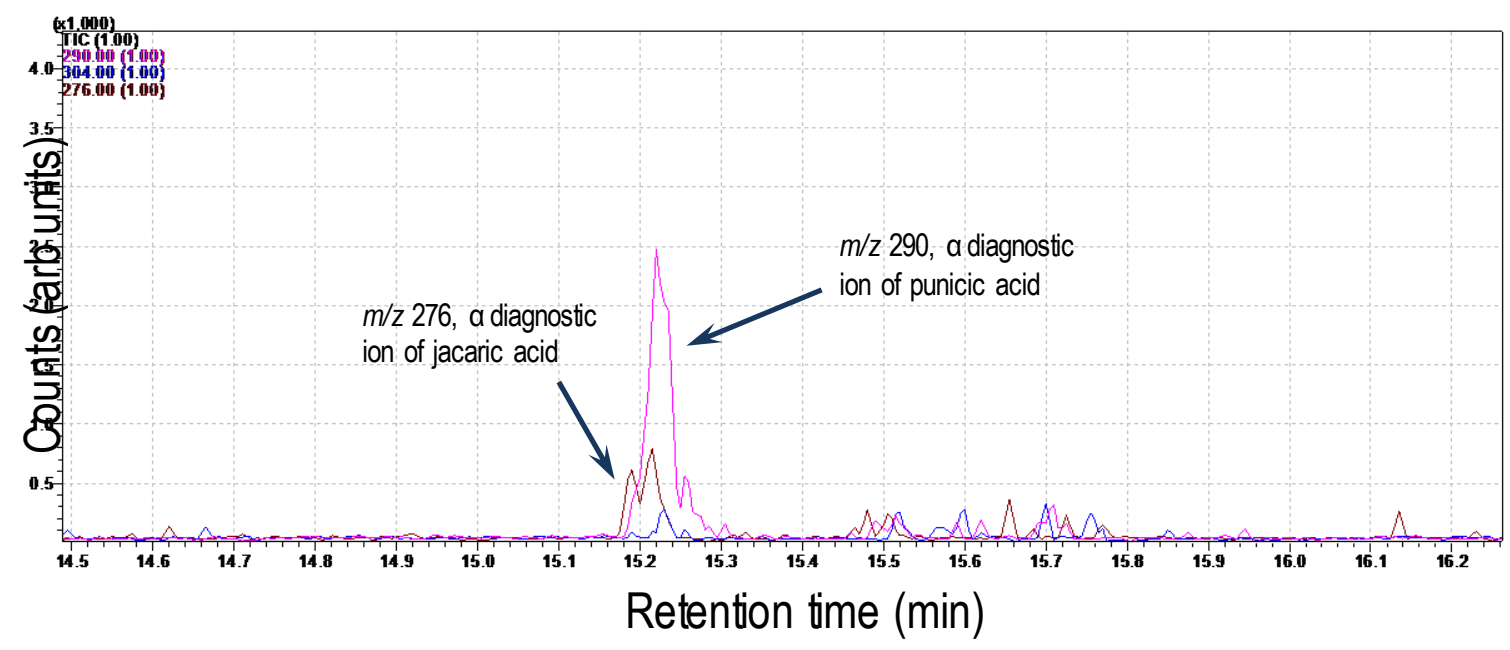

197 